\title{
Negative Pressure Wound Therapy and Skin Graft in Madura Foot Treatment
}

\author{
Ali Murat Kalender ${ }^{1}$, Halit Baykan², Fuat Özkan ${ }^{3}$, Harun Çıralık ${ }^{4}$ Perihan Öztürk ${ }^{5}$, Mustafa Gül ${ }^{6}$, Hasan Uçmak ${ }^{7}$ \\ ${ }^{1}$ Department of Orthopaedics and Traumatology, Faculty of Medicine, Kahramanmaraş Sütçü Imam University, \\ Kahramanmaraş, Turkey \\ ${ }^{2}$ Department of Plastic Reconstructive and Aesthetic Surgery, Faculty of Medicine, Kahramanmaraş Sütçü Imam University, \\ Kahramanmaraş, Turkey \\ ${ }^{3}$ Department of Radiology, Faculty of Medicine, Kahramanmaraş Sütçü Imam University, Kahramanmaraş, Turkey \\ ${ }^{4}$ Department of Pathology, Faculty of Medicine, Kahramanmaraş Sütçü Imam University, Kahramanmaraş, Turkey \\ ${ }^{5}$ Department of Dermatology, Faculty of Medicine, Kahramanmaraş Sütçü İmam University, Kahramanmaraş, Turkey \\ ${ }^{6}$ Department of Microbiology, Faculty of Medicine, Kahramanmaraş Sütçü İmam University, Kahramanmaraş, Turkey \\ ${ }^{7}$ Department of Infectious Disease, Faculty of Medicine, Kahramanmaraş Sütçü Imam University, Kahramanmaraş, Turkey
}

\begin{abstract}
Madura foot is a rare, loca, chronic granulomatous skin infection which commonly affects the adult male foot. Medical treatment reduces the size of the lesion but surgical excision is necessary for radical treatment. We present a case of a 59 year old male farmer diagnosed as actinomadura of the right foot treated with medical treatment, total excision, negative pressure wound therapy and split thickness skin graft.
\end{abstract}

Key Words: Madura foot, negative pressure wound therapy, split thickness skin graft

Received: 05.12.2011 Accepted: 16.02 .2012

\section{Introduction}

Madura foot or Mycetoma is termed a local chronic granulomatous infection of the skin and subcutaneous tissue after traumatic inoculation of the causative fungal (Eumycetoma) or bacterial (Actinomycetoma) organism (1). Classical clinical presentation includes gross swelling of the affected limb with multiple sinus tracts to the skin, draining pus, blood and fungal grains. Progression to bone invasion and destruction may occur with time (2). Herein we report a patient with Madura foot treated by medical treatment combined with total lesion excision, negative pressure wound therapy for wound granulation and split thickness skin graft.

\section{Case Report}

A 59-year-old farmer man was referred to the dermatologist by a general practitioner with a 10 year history of right ankle swelling and pus drOainage. He had lived in a village all his life in the Southern part of Turkey. He had visited several doctors since this swelling. The doctors had prescribed some drugs and referred him to specialist doctors. However, he did not take his medicine regularly or visit the specialists because the lesion was painless and he was not limping. In recent months however, he had felt right knee pain and started limping. The dermatologist sent us the patient with a Madura foot and right knee gonarthrosis diagnosis.

When we examined the patient, there was a large, soft tumour-like lesions over the dorsum of the right ankle.
The dimensions were $170-96 \mathrm{~mm}$. The patient did not remember any foot or ankle trauma. He described some yellow drainage (2-4 times in year) and very slow growth of the lesion (Figure 1). His ankle and foot ROM were normal. His standard standing $A P$ and lateral radiographies imaged normal bone structure. His ankle CT and MRI were also obtained.

MRI was made to characterize and evaluate the extent of the disease. T2-weighted fat-saturated and T1-weighted fat-saturated gadolinium enhanced MRI revealed a $9 \times 3 \mathrm{~cm}$ heterogenous mixture soft tissue mass involving subcutaneous tissue. In addition, multiple, small, distinct, spherical hyperintense lesions were noted, which were surrounded by low-intensity soft tissue. In the center of these lesions, there was a tiny hypointense focus, resulting in the dot-in-circle sign which is considered pathognomonic of mycetoma (Figure 2). The granulomatous lesion invaded the skin and subcutaneous tissue but not the vascular tissue, bone or tendons. There were no focal fluid collections at other sites.

His right knee was grade 2-3 gonarthrotic. He had suffered a violent knee trauma which was treated by a local bonesetter 4 years earlier. The knee MRI demonstrated complex degenerative lesions in both the medial and lateral meniscus and Anterior Cruciate Ligament rupture. The regional lymph nodes were hypertrophied.

Direct microscopic analysis and culture of the white-yellowish grains included gram staining, which showed extensively branched gram-positive hyphae less than $1 \mathrm{~mm}$ in diameter, allowing a diagnosis of Actinomycetoma to be made. The grains were placed onto plates containing blood agar 


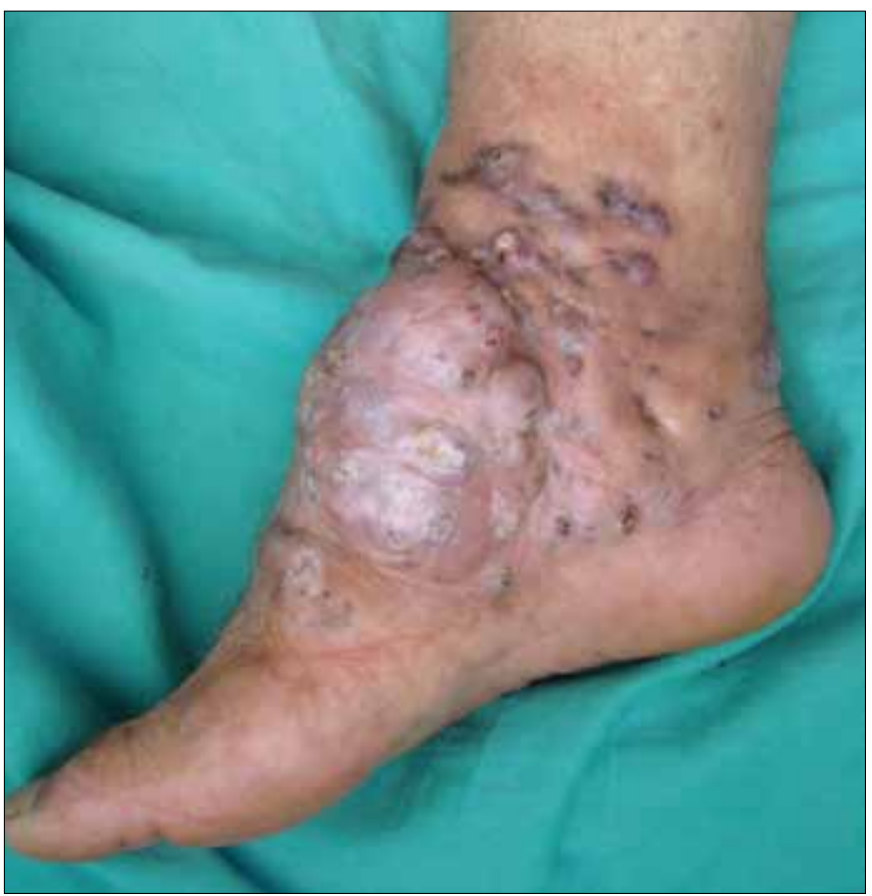

Figure 1. The medial aspect of the patient at the beginning of treatment

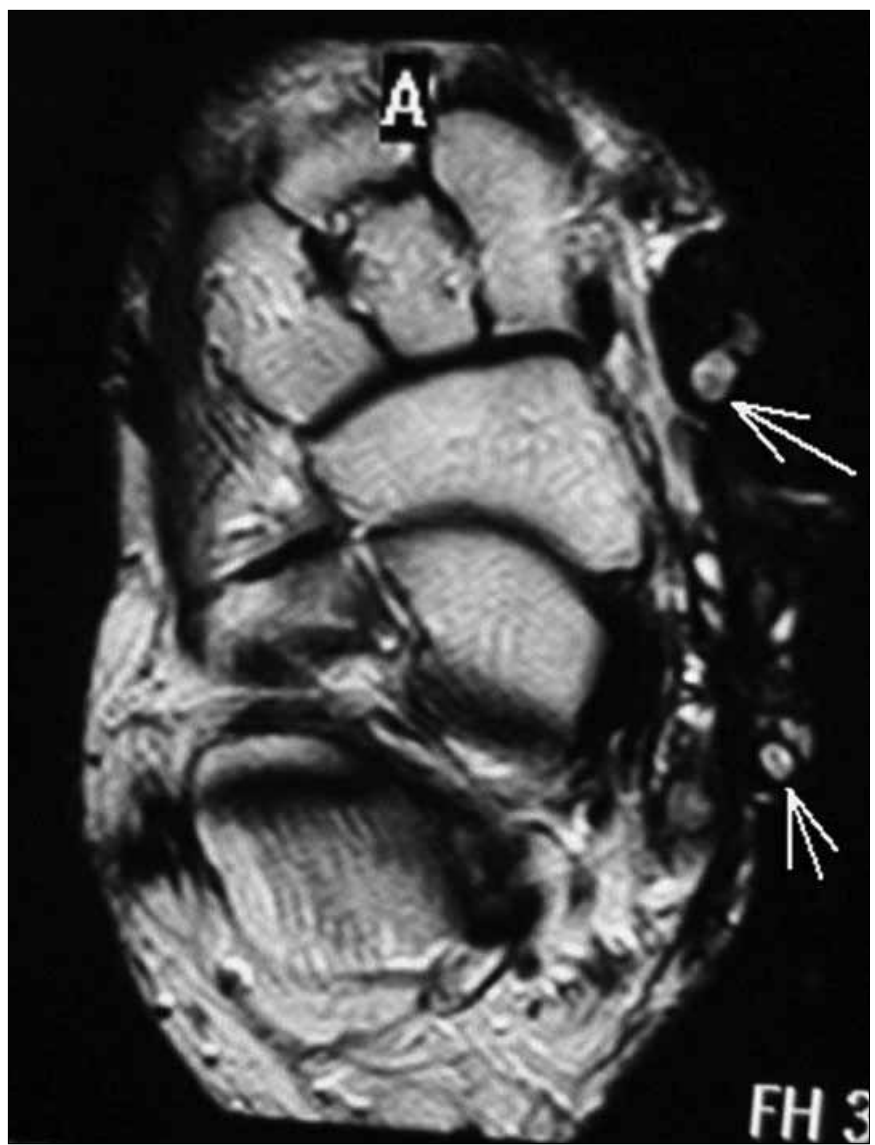

Figure 2. Spin echo-T2 axial image shows small spherical hyperintense lesions contain low signal central dot (dot-incircle sign) consistent with fungal grain surrounded by tissue of low signal intensity and incubated under aerobic and anaerobic conditions at $35^{\circ} \mathrm{C}$. Within 7 days, very small colonies, adherent, smooth, rough, rose coral or peach-coloured were isolated from the blood agar plates cultured under aerobic conditions. The Actinomadura madurae is seen in pus culture. We started medical treatment with oral $100 \mathrm{mg}$ tetracycline and $1 \mathrm{gr}$ IM streptomycin. After 3 months we prescribed $300 \mathrm{mg}$ oral rifampicin instead of streptomycin.

The lesion got softer and decreased to half size but did not resolve completely in 1 year of medical treatment. All regional lymph nodes were dissolved. A dermatology, plastic surgery and orthopaedic consultation agreed to a total surgical excision combined with medical treatment. We performed total excision then NPWT for wound granulation. The granulation was observed in 2 weeks (Figure 3). The wound was closed by split thickness skin graft. The graft was fully healed in 2 weeks (Figure 4). He was given TMP-SMX and tetracycline for 3 months after surgical excision. We did not observe any granulomatous tissue or drainage after surgery.

At surgery, a $16 \times 10 \mathrm{~cm}$ chronically inflamed mass over the ankle dorsum was excised, the cut surface showed yellowish illdefined nodules. It was sent for gram stain, Ziehl-Neelsen stain for mycobacteria; special stains for fungus (periodic acid-Schiff); anaerobic and aerobic culture; and for histological analysis. The tuberculosis culture, PCR and stain were negative.

Histopathologic examination of the tissue showed that actinomycotic colonies with mixed inflammation, hemosiderin containing macrophages, neutrophils, plasma cells and lymphocytes surrounded by collagenised dense fibrous tissue in the papillary and reticular dermis. The filaments and hyphaes in these colonies gave a positive reaction with Gomori methanemin silver and periodic acid-Schiff dyes. The malignant transformation, granulamatous reaction and giant cells were not observed in histopathologic examination. These findings were consistent with actinomyces.

The patient was followed for 2 months after surgical treatment. His ankle healed without any drainage or granulomatous lesion. All the liver and kidney enzymes were normal during follow up. When we began treatment, we obtained written informed consent for surgery and publication. He did not visit us for the $3^{\text {th }}$ month control. We called to invite him for a follow up control. Unfortunately, we learned that he had died at home in his sleep with anterior myocardial infarction. Thus we could not take a late photograph of the healed lesion.

\section{Discussion}

Mycetoma is a serious health problem in many tropical and sub-tropical regions. Recent case reports showed that it can be seen almost all over world, but this is extremely uneven. Painless subcutaneous swelling, sinus tract formation and discharge that contain grains are pathognomonic of mycetoma (3). Usually patients visit doctors at very late stages due to socio-economic reasons. Our patient had lived 10 years with this classical history of painless ankle lesion before visiting a dermatologist and an orthopaedic surgeon.

The organisms are present in the soil in the form of grains. Traumatic inoculation of the subcutaneous tissue caused by sharp objects such as thorn pricks, or splinters is thought to be the route of entry (4). However; this theory has been recently 


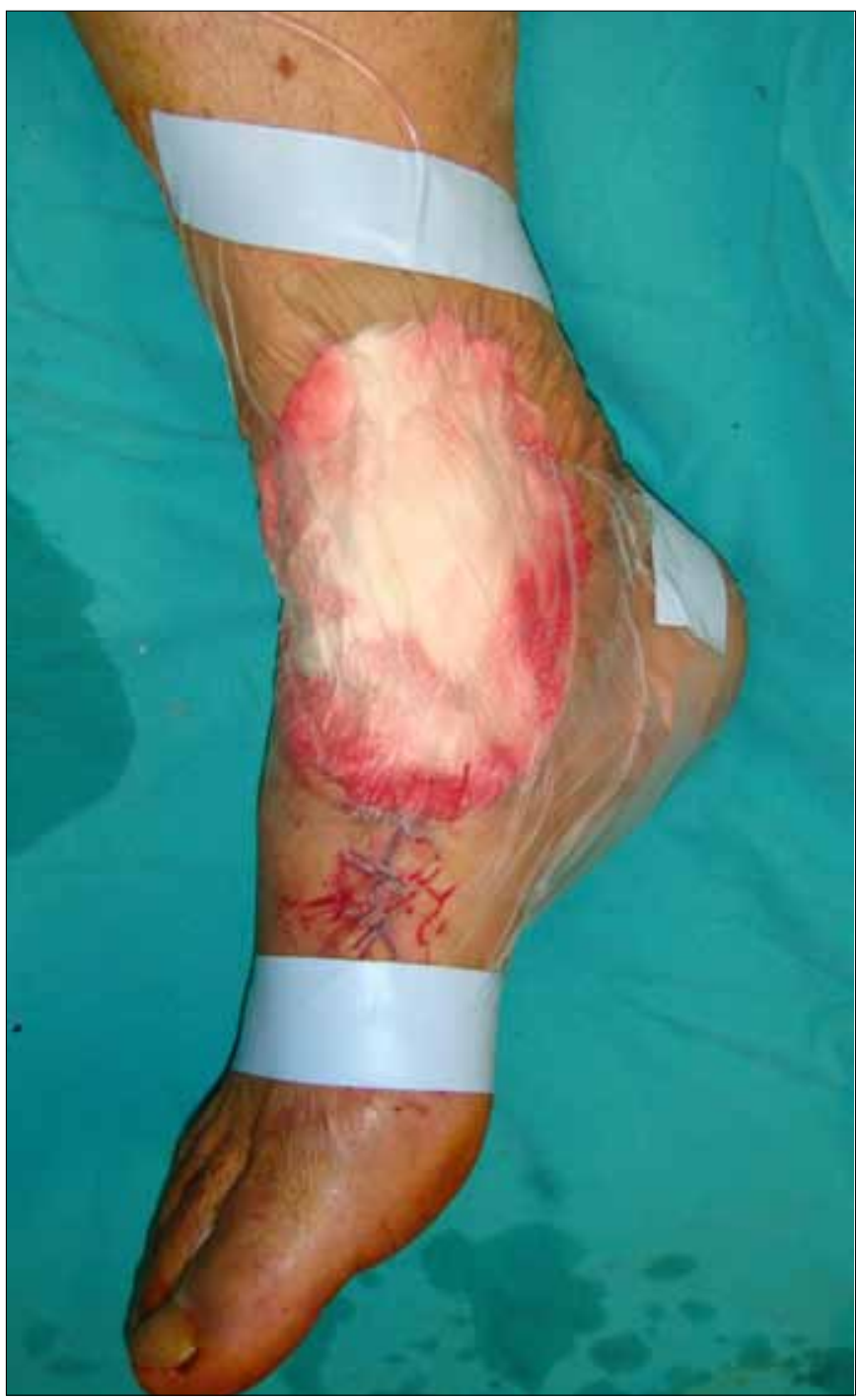

Figure 3. The negative pressure wound therapy (NPWT) application just after excision

disputed, as many patients have no history of trauma at the infection site. In areas where mycetoma is frequent, the habit of going barefoot is common and thorns are plentiful and as a result, natural infection is expected to be more frequent than it actually is. The soles of the feet are not a commonly affected site, as in our patient (4).

The mycetoma commonly affects adult males between 20 40 years of age and these are the most active members of society, especially in underdeveloped countries (5). Mycetoma is seen generally in farmers, field workers and in herdsmen, and in endemic areas, people of other occupations are also affected. Our patient was a farmer and sometimes worked barefoot.

The foot is affected most frequently ( $80 \%$ of cases) in mycetoma (6). The lesions are seen commonly on the dorsal aspect of the forefoot and, for unexplained reasons, the left foot is affected more than the right. The infection usually remains localised and constitutional disturbances are rare but, when they do occur, they are generally due to septiacemia or to immunosuppression (6). The right ankle dorsum was involved

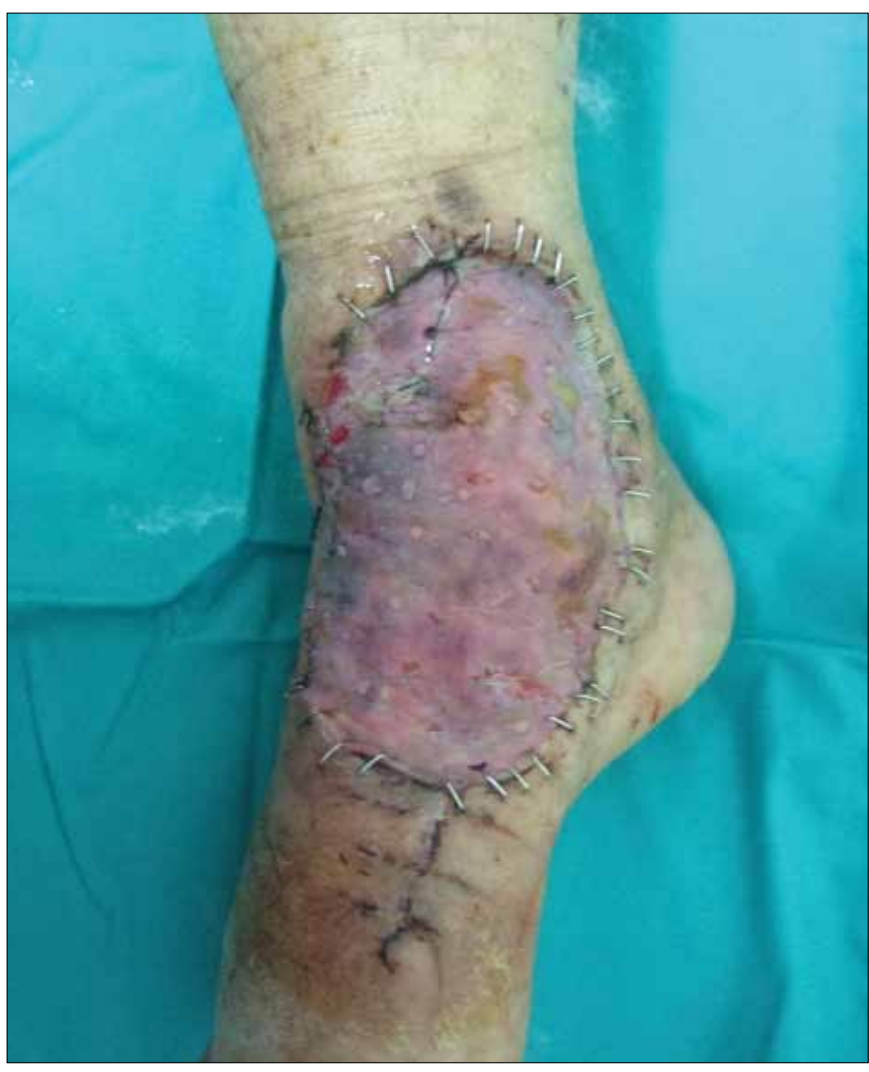

Figure 4. The picture of healed split thickness skin graft

in our patient. The nerves, tendons and bones are rarely affected until late in the disease course. These structures were not involved in our patient. In about $1-3 \%$ of cases there is lymphatic spread to the regional lymph nodes. During the active phase of the disease these regional lymphatic foci may suppurate and discharge as well. Malnutrition and anaemia may be seen in late mycetoma (6). It can be fatal, especially if it affects the skull (7). We observed lymph node hypertrophy in our patient, which were dissolved after medical treatment.

McElroy et al. (8) described the three cardinal features associated with Madura foot: tumefaction, formation of sinus tracts, and the presence of grains in the affected tissue. The initial symptoms often go unnoticed by the patient or arise as a small bump. The most common presentation is a painless lump or a swollen foot. Eventually, multiple draining sinuses will develop with the discharge of pus and granules. Classically, a cycle develops in which old sinuses heal and new sinuses form, causing the progressive development of granulation and scar tissue that can cause deformity or disfigurement (9). All these pathological findings were present in our patient.

The most accurate diagnosis of Mycetoma is made by fine needle aspiration cytology and culture (10). Mycetoma lesion has a distinct appearance in a cytology smear and is characterised by the presence of polymorphous inflammatory cells consisting of an admixture of neutrophils, lymphocytes, plasma cells, histiocytes, macrophages and foreign body giant cells and grains. In sections, the grain is closely surrounded by, and occasionally infiltrated by neutrophils causing its fragmentation. Outside the neutrophil zone, monocytic cells and giant cells are seen. Grains can be seen histologically as "sulphur granules" surrounded by polymorphonuclear cells form- 
ing the purulent tissue reaction. The filaments and hyphae of the causal agent can be stained better in biopsy samples with Gram stain, Gomori methenamine silver or periodic acid-Schiff stains. Purulent tissue is surrounded by granulation tissue rich in fibroblasts. This granulation tissue protects microorganisms from given antibiotherapy. Thus surgical excision is needed usually as in our patient.

Combined drug therapy is preferred to a single drug to avoid drug resistance and to eradicate residual infection. The common drugs in use include combination of streptomycin sulphate (14 mg/kg daily) and diaminodiphenyl sulphone (dapsone) $(1.5 \mathrm{mg} / \mathrm{kg}$ twice daily) (11). If there is no response for a few months or if there is a persistent side effect then dapsone is replaced by co-trimoxazole (14 mg/kg twice daily). An excellent therapeutic response to amikacin sulphate alone or in combination with co-trimoxazole has been reported (11). The combination of medical treatment before and after surgery results in the most successful outcome (12). We performed total excision with antibiotic combination since bones, tendons and vascular structures were intact.

The duration of treatment varies with the severity of the infection and the general health status of individual patients. Treatment may need to continue for years and the liver function of the patients needs regular monitoring (13). Therefore, we regularly checked the liver and kidney function tests every month in our patient.

NPWT, based on application of subatmospheric pressure, has revolutionized the management of wounds (14). It has been successfully used in situations of wounds complicated by the presence of burn, infection, poor circulation, exposed bone or artificial implants, or previous wound dehiscence (15). NPWT facilitates healing by reportedly improving the rate of angiogenesis, endothelial proliferation, the integrity of the capillary basement membrane, capillary blood flow, capillary caliber, and by decreasing interstitial edema and bacterial burden within the wound (16). After excision of the lesions and NPWT application, we observed good granulation over tendons and extensor retinaculum in our patient. We inspected the wound closely for any discharge and haemorrhage after surgery but they were not observed.

NPWT is being used in an increasing number of clinical indications. It is used in the treatment of graft infections, complex chronic abdominal wounds, in order to promote the formation of granulation tissue in a variety of clinical situations involving exposed orthopaedic implants, enterocutaneous and enteroatmospheric fistulae, diabetic foot, sternal wounds, non-healing extremity wounds, pressure ulcers, large infected wounds of the face, and difficult perineal and genital wounds (14). To our knowledge there is no article related with Madura foot and NPWT in English literature.

Wounds secondary to trauma, burns and various surgical procedures and chronic medical conditions such as venous stasis and diabetes mellitus have all been traditionally closed with skin grafting techniques (17). The wound granulation was seen in 15 days and then a split thickness skin graft was applied. The graft healed fully. We planned to take late photographs of the healed foot but the patient died with a sudden myocardial infarction. His son told us that there was no mass or pain around the ankle and the patient was grateful for all his treatment.

The dot-in-circle sign is a recently identified sign showing the exclusive pathological feature of mycetoma. Sarris et al. described this sign as a small hypointense focus within highintensity spherical lesions (18). The central low signal "dot" corresponded histological to the fungal balls or grains and was contained within a high-signal granuloma.

\section{Conclusion}

The medical treatment alone is not sufficient for Madura foot. A long term drug application is necessary and the patient must be monitered closely for liver and kidney functions. Total excision should be done if possible. The negative pressure wound therapy is a good choice for granulation and wound care. Skin graft can be used for closure of defects in Madura foot in a standard fashion.

\section{Conflict of Interest}

No conflict of interest was declared by the authors.

\section{References}

1. Ahmed AO, van Leeuwen W, Fahal A, van de Sande W, Verbrugh $\mathrm{H}$, van Belkum A. Mycetoma caused by madurella mycetomatis: a neglected infectious burden. Lancet 2004;4:566-74. [CrossRef]

2. Burkus JK. Primary mycetoma infection of the distal tibia secondary to an occult foreign body. Foot Ankle 1985;6:47-52.

3. Fahal AH, Hassan MA. Mycetoma Br J Surg 1992;79:1138-41. [CrossRef]

4. Ahmed A, Adelmann D, Fahal AH, Verbrugh $H$, van Belkum A, de Hoog S. Environmental occurrence of Madurella mycetomatis, the major agent of human eumycetoma in Sudan. J Clin Microbiol 2002;40:1031-6. [CrossRef]

5. Fahal AH, Suliman SH, Gadir AFA, El Hag IA, El Amin FI, Gumaa $\mathrm{SA}$, et al. Abdominal wall mycetoma: unusual presentation. Trans. R Soc Trop Med Hyg 1994;88:78-80. [CrossRef]

6. Fahal AH. Mycetoma: a thorn in the flesh. Trans R Soc Trop Med Hyg 2004;98:3-11. [CrossRef]

7. Gumaa SA, Mahgoub ES, El Sid MA. Mycetoma of the head and neck. Am J Trop Med Hyg 1986;35:596-600.

8. McElroy JA, Prestes CD, Su WP. Mycetoma: infection with tumefaction, draining sinuses, and "grains." Cutis 1992;49:107-10.

9. Young BA, Fee MJ, Giacopelli JA, Granoff DP, Kobayashi W. Mycetoma J Am Podiatr Med Assoc 2000;90:81-4.

10. El Hag IA, Fahal AH, Khalil EAG. Fine nee-dle aspiration cytology of mycetoma. Acta Cytol 1996;40:461-4. [CrossRef]

11. Mahgoub ES. Medical treatment of mycetoma in the Sudan. Sudan Med J 1994;32:88-97.

12. Ahmed AO, van de Sande WJ, Verbrugh $H$, Fahal A, van Belkum A. Madurella mycetomatis strains from mycetoma lesions in Sudanese patients are clonal. J Clin Microbiol 2003;41:4537-41. [CrossRef]

13. Ahmed AO, van Leeuwen $W$, Fahal $A$, van de Sande $W$, Verbrugh $H$, van Belkum A. Mycetoma caused by Madurella mycetomatis: a neglected infectious burden. Lancet infect Dis 2004;4:566-74. [CrossRef]

14. Cipolla J, Baillie DR, Steinberg SM, Martin ND, Jaik NP, Lukaszczyk JJ, et al. Negative pressure wound therapy: Unusual and innovative applications. OPUS 12 Scientist 2008;2:15-29.

15. Van der Velde M, Hudson DA. VADER (vacuum-assisted dermal recruitment): a new method of wound closure. Ann Plast Surg 2005;55:660-4. [CrossRef]

16. Weed T, Ratliff C, Drake DB. Quantifying bacterial bioburden during negative pressure wound therapy: does the wound VAC enhance bacterial clearance? Ann Plast Surg 2004; 52:279-80. [CrossRef]

17. Jones JE, Nelson EA. Skin grafting for venous leg ulcers. Cochrane Database Syst Rev 2007;18:CD001737.

18. Sarris I, Berendt AR, Athansous N, Ostlere SJ. MRI of mycetoma of the foot: two cases demonstrating the dot-in-circle sign. Skeletal Radiol 2003;32:179-83. 